\title{
Does the bromocriptine-rebound method improve embryo quality?
}

\author{
Naoko Moride, Akira Kuwahara, Mizuho Yamashita, Yu Tanaka, Toshiya Matsuzaki, \\ Toshiyuki Yasui, and Minoru Irahara \\ Department of Obstetrics and Gynecology, Institute of Health Biosciences, the University of Tokushima \\ Graduate School Tokushima, Japan
}

\begin{abstract}
Aim : To examine whether the bromocriptine-rebound (BR) method improves pregnancy outcomes after previous unsuccessful assisted reproductive technology (ART) attempts. Patients/study design : In this study we retrospectively analyzed data from a total of 121 women with normal serum prolactin (PRL) levels and a history of repeated unsuccessful ART procedures. Pregnancy outcomes and hormonal data were compared between the long protocol and BR method. Both procedures were similar, except that in the BR method, bromocriptine was administered daily from day 5 of the preceding cycle until 7 days before ovarian stimulation. Results : The number of fertilized oocytes, cleaved embryos and transplant embryos were significantly higher with the BR method than with the long protocol even though the numbers of retrieved oocyte were same in both groups. The ratio of the good embryos, the clinical pregnancy rate was higher with the BR method than with the long protocol. The embryo score with the BR method were significantly higher than that with the long protocol. Conclusion : BR method could provide the better embryos and improve the transplantation rate in women with previous unsuccessful ART attempts J. Med. Invest. 58 : 63-66, February, 2011
\end{abstract}

Keywords : bromocriptine, prolactin, ovarian stimulation, pregnancy rate

\section{INTRODUCTION}

Through the development of assisted reproductive technology (ART), in vitro fertilization and embryo transfer (IVF-ET) and intracytoplasmic sperm injection (ICSI) have assumed central roles in the treatment of infertility. However, numerous patients do not conceive even after several cycles of ART. Possible causes of failure after repeated attempts with ART include increasing tendency of getting married at a late age, poor response to ovulation induction,

Received for publication September 27, 2010 ; accepted November $11,2010$.

Address correspondence and reprint requests to Akira Kuwahara, $\mathrm{MD}, \mathrm{PhD}$, Department of Obstetrics \& Gynecology, Institute of Health Biosciences, the University of Tokushima, 3-18-15 Kuramoto, Tokushima 770-8503, Japan and Fax : +81-88-6312630. problems with embryo implantation, and male factors. Various trials have been accomplished for poor responder in each institution. Jinno et al proposed that patients with poor IVF outcomes using the long protocol may have low responsiveness of granulose cell to PRL (1). They proposed the bromocriptinerebound (BR) method as a possible treatment options (2). The aim of this study was to examine whether the BR method improved pregnancy outcomes in women with previous unsuccessful ART attempts.

\section{PATIENTS AND METHODS}

Patients and study design :

In this study we retrospectively analyzed data 
from a total of 121 women with normal serum prolactin (PRL) levels and a history of repeated unsuccessful IVF or ICSI procedures at the University of Tokushima from March 1998 to October 2000. IVF/ICSI outcomes and hormonal data were compared between 99 woman with the long protocol and 22 women in the BR method. Patient background was shown in Table 1.

Table 1. Patient background

\begin{tabular}{lcc} 
& $\begin{array}{c}\text { Long } \\
\text { protocol } \\
(\mathrm{n}=99)\end{array}$ & $\begin{array}{c}\text { Bromocriptine } \\
\text { rebound method } \\
(\mathrm{n}=22)\end{array}$ \\
\hline Average age(y) & $35.1 \pm 4.3$ & $35.4 \pm 3.7$ \\
$\mathrm{LH}(\mathrm{mIU} / \mathrm{ml})$ & $4.7 \pm 2.4$ & $4.8 \pm 2.7$ \\
$\mathrm{FSH}(\mathrm{mIU} / \mathrm{ml})$ & $7.9 \pm 2.5$ & $7.9 \pm 3.0$ \\
$\mathrm{PRL}(\mathrm{ng} / \mathrm{ml})$ & $7.5 \pm 8.3$ & $5.9 \pm 3.3$
\end{tabular}

Values are presented as the mean \pm S.D.

\section{Ovarian stimulation regimens :}

In the long protocol, we initiated administration of buserelin acetate (600 micro g/day ; Suprecur, Hoechst, Tokyo, Japan) on the $7^{\text {th }}$ day of high phase of basal body temperature before treatment. After menstruation started, ovarian stimulation was started with 150 IU of follicle-stimulating hormone (FSH ; Fertinom $\mathrm{P} \circledast$, Serono, Tokyo) for 4 days and then $225 \mathrm{U}$ of human menopausal gonadotropin (HMG ;
Humegon ${ }^{\circledR}$, Organon, Osaka, Japan) for 5 days, followed by administration of human chorionic gonadotropin (hCG) 5,000 IU. Oocytes were collected 36 $\mathrm{h}$ after hCG administration. In the BR method, bromocriptine $\left(2.5 \mathrm{mg} /\right.$ day, Teluron ${ }^{\circledR}$, Bayer pharma, Osaka, Japan) was administered orally daily from day 5 of the preceding cycle until 7 days before ovarian stimulation (Figure 1).

\section{IVF-ET procedure :}

Motile spermatozoa were collected by the swimup technique. Oocytes were cultured in human tubal fluid medium (Irvine Science, Santa Ana, CA, USA), to which $10 \%$ of the patient's serum was added for 2-4 h, and inseminated at a concentration of $50,000 / \mathrm{ml}$ motile spermatozoa. Oocytes were considered to be fertilized when 2 pronuclei were observed 14-16 h after insemination. Embryos were transferred $40-48 \mathrm{~h}$ after insemination. At $38-40 \mathrm{~h}$ after insemination, we evaluated embryos according to the criteria shown in Table 2. Embryo transfer

Table 2 Embryo evaluation method at the University of Tokushima

\begin{tabular}{|c|c|c|c|c|}
\hline Point & 0 & 2 & 3 & 5 \\
\hline $\begin{array}{l}\text { The number of the } \\
\text { blastomeres }\end{array}$ & 1 & - & $2 \sim 3$ & $4 \leqq$ \\
\hline Fragmentation (\%) & $\geqq 50$ & $25 \leqq<50$ & $<25$ & 0 \\
\hline $\begin{array}{l}\text { Demerit mark item } \\
-2 \text { points }\end{array}$ & \multicolumn{4}{|c|}{$\begin{array}{l}\text { Zona pellucida transformation, Cytoplasm } \\
\text { black Vacuole denaturation, Blastomere big } \\
\text { things and small things inequality }\end{array}$} \\
\hline
\end{tabular}

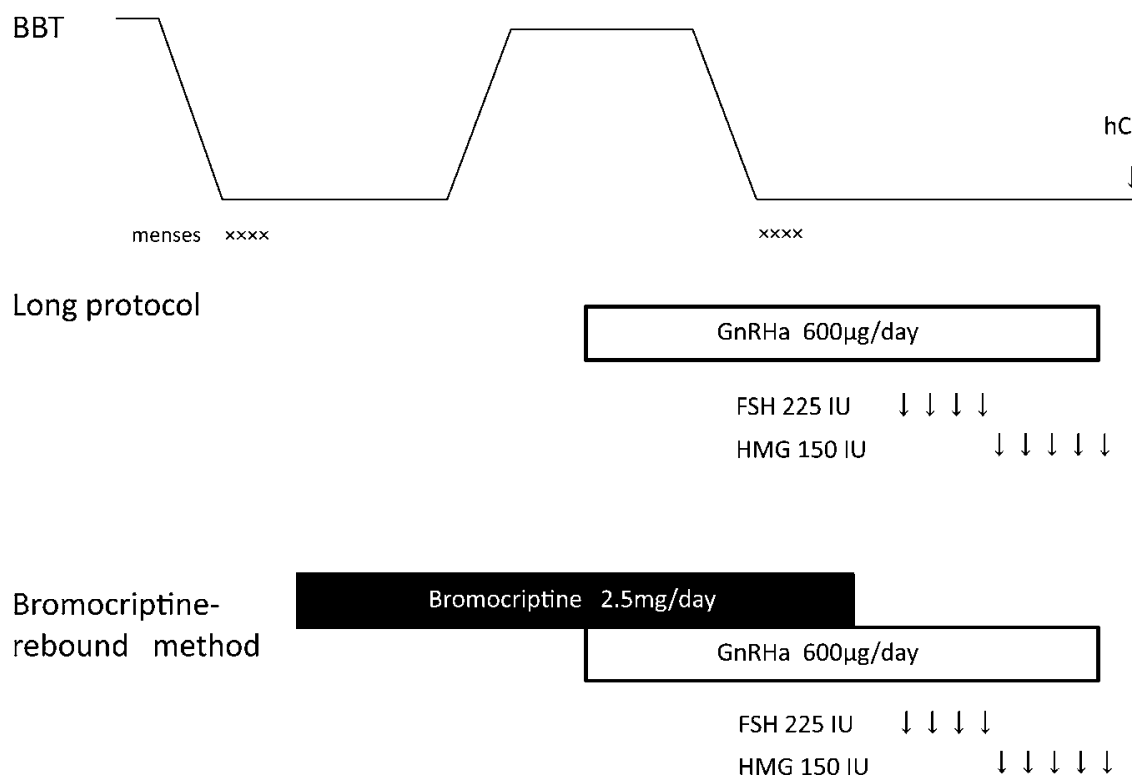

Figure 1 Ovarian stimulation regimens 
of 1-3 embryos was performed according to the recommendation from the Japan Society of Obstetrics and Gynecology. We administered hCG 1,500 U/day 3 times every $3^{\text {rd }}$ day from the embryo transfer day.

\section{Statistical analysis :}

Data were analyzed using Student's $t$-test and the chi-square test. $\mathrm{P}<0.05$ was considered statistically significant. Results are presented as mean \pm S.D. unless otherwise stated.

\section{RESULTS}

The peak value of estradiol was $2,125 \mathrm{pg} / \mathrm{ml}$ with the BR method and $1,790 \mathrm{pg} / \mathrm{ml}$ with the long protocol, and the differences were not significant (Figure 2). The results of the long protocol and the BR method are summarized in Table 3 . The number of fertilized oocytes, cleaved embryos and transplant embryos were significantly higher with the BR method than with the long protocol $(4.0 \pm 3.5 \mathrm{vs}$. $5.4 \pm 3.7,3.8 \pm 3.3$ vs. $5.0 \pm 3.6,2.3 \pm 1.1,2.7 \pm 1.0$, respectively ; $\mathrm{P}<0.05)$ even though no significant differences were observed in the number of retrieved oocytes between the long protocol and BR method. The clinical pregnancy rate was significantly higher with the BR method than with the long protocol. The mean embryo score with the BR method was significantly higher than that with the long protocol (6.0 $\pm 1.4,5.2 \pm 2.5$, respectively ; $\mathrm{P}<0.05$ ) (deta was not shown). The ratio of the good embryos ( $>6$ points) was higher with the BR method than with the long protocol $(\mathrm{P}<0.05)$.

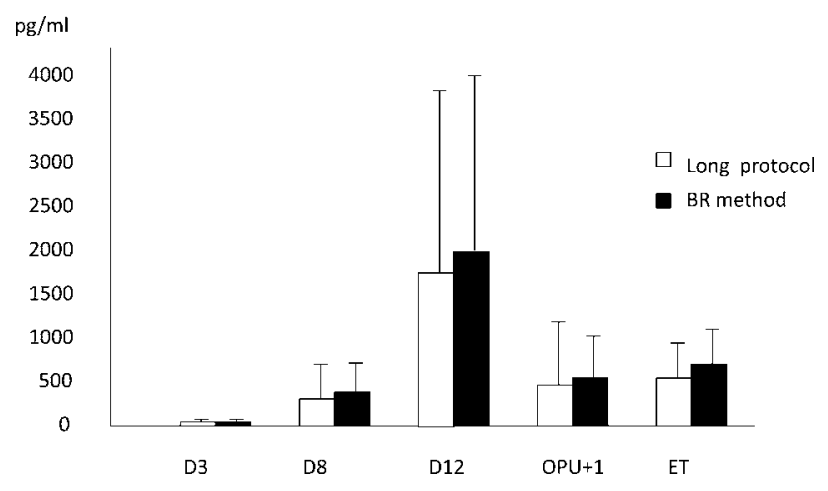

Figure 2 Serum estradiol concentration

Estradiol levels are higher with the BR method compared to those with the long protocol, but the differences are not significant. Data were analyzed by unpaired $t$ test.
Table. 3 In vitro fertilization outcomes for long protocol and Bromocriptin-rebound method

\begin{tabular}{lll} 
& $\begin{array}{c}\text { Long } \\
\text { protocol } \\
(\mathrm{n}=99)\end{array}$ & $\begin{array}{c}\text { Bromocriptin } \\
\text { rebound method } \\
(\mathrm{n}=22)\end{array}$ \\
\hline Retrieved oocytes & $7.5 \pm 4.9$ & $8.2 \pm 5.0$ \\
Fertilized oocytes & $4.0 \pm 3.5$ & $5.4 \pm 3.7^{\mathrm{a}}$ \\
Cleaves embryos & $3.8 \pm 3.3$ & $5.0 \pm 3.6^{\mathrm{a}}$ \\
Transplant embryos & $2.3 \pm 1.1$ & $2.7 \pm 1.0^{\mathrm{a}}$ \\
Clinical pregnancies & $10.1 \%(10 / 99)$ & $27.2 \%(6 / 22)^{\mathrm{b}}$ \\
\hline Total embryo quality & $24.4 \%(180 / 738)$ & $32.8 \%(59 / 180)^{\mathrm{b}}$
\end{tabular}

Values are presented as mean \pm S.D.

*Good embryo : $>6$ points in Tokushima University's criteria aP $<0.05$ compared with the long protocol ( $t$-test, unpaired) ${ }^{b} \mathrm{P}<0.05$ compared with the long protocol $\left(\chi^{2}\right.$-test $)$

\section{DISCUSSION}

In our study, morphologically better embryos can be transplanted using the BR method in woman with previous failed ART attempts. PRL is known to play a significant role in regulating ovarian functions, including folliculogenesis, steroid genesis, ovulation, and corpus lutenum function $(3,4)$. PRL levels are generally high in patients with ovulation and steroidogenesis disorders. It is reported that in the hypoprolactinemia, the rate of cleavage embryo and pregnancy rate was low. In addition, serum PRL levels after hCG administration are higher in pregnant women than in non-pregnant women after IVF (5). In a study of rabbit oocytes, addition of PRL to the culture fluid was found to promote oocyte maturity (6).

The serum PRL levels with the BR method continuously increased 4 days after the end of bromocriptine administration to the day hCG was administered (2). A follicular PRL receptor has been reported in connection with human IVF $(3,7,8)$, and a high concentration of PRL in the follicular fluid was associated with maturation of the oocytecumulus complex, resulting in successful fertilization and pregnancy (9). Jinno et al. proposed that the granulosa cells in patients with poor IVF outcomes after the long protocol may show low responsiveness to PRL, possibly in the postreceptor pathway, thereby causing compensatory increases in the levels of serum PRL. They suggested that in such patients, the BR method probably improves oocyte maturation by restoring postreceptor responsiveness of the granulosa cells to PRL during the hypoprolactinemic period and increasing the serum PRL concentration through a rebound phenomenon after 
discontinuation of bromocriptine (2).

In our study, serum PRL levels in BR method and long protocol showed no significant difference. However, the embryo quality was significantly improved in cases with BR method. Application of the BR method in women with previous unsuccessful ART attempts appears to increase serum PRL values during ovarian stimulation and promote oocyte maturation through the presence of PRL receptors in the granulosa cells even in normal PRL patients. An increase in the number of normal embryos can be expected to result in improved pregnancy outcomes (10). These factors increase the fertility rate and improve embryo quality, but the underlying mechanism is still unclear.

In conclusion, the BR method could provide the better quality in retrieved oocytes and improve embryo quality and number of developing embryos, then improve the transplantation rate in women with previous unsuccessful ART attempts

\section{REFERENCES}

1. Jinno M, Yoshimura Y, Ubukata Y, Nakamura $\mathrm{Y}$ : A novel method of ovarian stimulation for in vitro fertilization : bromocriptine-rebound method. Fertil Steril 66(2) : 271-274, 1996

2. Jinno M, Katsumata Y, Hoshiai T, Nakamura Y, Matsumoto K, Yoshimura Y : A therapeutic role of prolactin supplementation in ovarian stimulation for in vitro fertilization : The bromocriptine-rebound method. J Clin Endocrinol Metab 82(11) : 3603-3611, 1997

3. McNeilly AS : Prolactin and ovarian function.
In : Muller EE, MacLeod RM, eds. Neuroendocrine perspectives. Elsevier, Amsterdam, 1984, pp.279-316

4. Oda T, Yoshimura Y, Takehara Y, Kohriyama S, Sano Y, Tanabe K, Kobayashi T, Nakamura $\mathrm{Y}$, Ohno T, Nozawa S : Effects of prolactin on fertilization and cleavage of human oocytes. Horm Res 35 : 33-38, 1991

5. Gonen Y, Casper RF : Does transient hyperprolactinemia during ovarian hyperstimulation interfere with conception or pregnancy outcome? Fertil Steril 51(6) : 1007-1010, 1989

6. Yoshimura Y, Nakamura Y, Yamada H, Ando M, Ubukata Y, Oda T, Suzuki M : Possible contribution of prolactin in the process of ovulation and oocyte maturation. Horm Res 35 : 22-32, 1991

7. Ben-David M, Schenker JG. Human ovarian receptors to human prolactin: Implications in infertility. Fertil Steril 38(2) : 182-186, 1982

8. Phelps JY, Bugg EM, Shamblott MJ, Vlahos NP, Whelan J, Zacur HA : Prolactin gene expression in human ovarian follicular cells. Fertil Steril 79(1) : 182-185, 2003

9. Laufer N, Botero-Ruiz W, DeCherney AH, Haseltine F, Polan ML, Behrman HR : Gonadotropin and prolactin levels in follicular fluid of human ova successfully fertilized in vitro. J Clin Endocrinol Metab 58(3) : 430-434, 1984

10. Hill GA, Freeman M, Bastias MC, Rogers BJ, Herbert CM 3rd, Osteen KG, Wentz AC : The influence of oocyte maturity and embryo quality on pregnancy rate in a program for in vitro fertilization-embryo transfer. Fertil Steril 52(5) : 801-806, 1989 